\title{
Avaliação da atividade antiinflamatória e analgésica do extrato bruto hidroalcoólico do zoantídeo Palythoa caribaeorum (Duchassaing \& Michelotti, 1860)
}

\author{
Carolina L.S. Soares ${ }^{1}$, Carlos D. Pérez ${ }^{1 *}$, Maria B.S. Maia ${ }^{2}$, Rejane S. Silva ${ }^{2}$, Liany F.A. Melo ${ }^{1}$ \\ ${ }^{1}$ Núcleo de Biologia - CAV, Universidade Federal de Pernambuco, 55608-680, Vitória de Santo Antão, PE, Brasil, \\ ${ }^{2}$ Laboratório de Farmacologia de Produtos Bioativos, Departamento de Fisiologia e Farmacologia, Universidade \\ Federal de Pernambuco, 50670-420, Recife, PE, Brasil
}

\begin{abstract}
RESUMO: O presente trabalho representa a primeira análise das atividades analgésica e antiinflamatória do extrato bruto hidroalcoólico do zoantídeo Palythoa caribaeorum. Foram realizados os testes de Writhing induzido por ácido acético $\left(1 \%, 10 \mathrm{~mL} \cdot \mathrm{kg}^{-1}\right.$ i.p.) e da chapa quente em camundongos para avaliar o efeito analgésico; e o teste de edema de pata de rata induzido por carragenina para avaliar a atividade antiinflamatória. As análises evidenciaram atividade analgésica no teste de Writhing no grupo tratado com $200 \mathrm{mg} . \mathrm{kg}^{-1}$ v.o. de extrato, com uma inibição de $47,22 \%$ do número de contorções abdominais ("Writhings"), revelando atividade estatisticamente significativa.
\end{abstract}

Unitermos: Palythoa caribaeorum, Zoantharia, Anthozoa, atividade antiinflamatória, atividade analgésica.

\begin{abstract}
Evaluation of the anti-inflammatory and analgesic activity of the crude hydroalcoholic extract of the zoanthid Palythoa caribaeorum (Duchassaing \& Michelotti, 1860)". This work represents the first pharmacological analysis on the crude hydroalcoholic extract of Palythoa caribaeorum. The analgesic activity was assayed in acetic acid-induced writhing test and with the hot-plate test with mice; while the anti-inflammatory activity was evaluated in carrageenan-induced hind paw edema in the rat. The analysis suggested an analgesic activity in the Writhing test on the group treated with the crude extract (200 mg. $\mathrm{kg}^{-1}$ v.o.) with an abdominal contortion's inhibition of $47,22 \%$.
\end{abstract}

Keywords: Palythoa caribaeorum, Zoantharia, Anthozoa, anti-inflammatory activity, analgesic activity

\section{INTRODUÇÃO}

Os cnidários antozoários são um grupo de organismos coloniais ou solitários, que apresentam uma grande variedade de formas, tamanhos e cores, habitando todos os mares do mundo, desde zonas costeiras até abissais (Pérez, 1999). Os zoantídeos são cnidários polipóides exclusivamente marinhos e habitantes dos recifes costeiros. Palythoa é um gênero de zoantídeos coloniais cujas espécies se caracterizam pela incorporação de grão de sedimento nos tecidos da sua parede corporal. $\mathrm{Na}$ espécie Palythoa caribaeorum (Duchassaing \& Michelotti, 1860) os pólipos estão conectados por um espesso tecido chamado cenénquima, o qual também agrega partículas na sua superfície. Estas colônias formam extensos "tapetes" localizados nas áreas submersas, mas quando estão expostos durante a maré baixa, seus pólipos produzem um muco que protege a colônia da dessecação. O muco da o nome popular de "baba-de-boi". Este zoantídeo é o mais abundante do litoral pernambucano, ocupando extensas regiões sobre os recifes.
O estudo químico com cnidários teve grande impulso durante a década do 60 com o descobrimento das prostaglandinas em varias espécies de corais moles do Caribe (em especial a gorgónia Plexaura homomalla) (Weinheimer; Spraggins, 1969). A extrema diversidade dos efeitos biológicos das prostaglandinas provocou, naquele momento, a coleta indiscriminada destas espécies, pondo em perigo as populações das mesmas em varias regiões caribenhas. Felizmente o desenvolvimento de métodos de síntese em laboratório avançou rapidamente, resguardando estes corais (Correia et al., 2002). Desde então, ficou em evidencia o potencial destes organismos como produtores de compostos de interesse biológico e farmacológico. A maior quantidade de metabólitos secundários de cnidários foi isolada de octocorais (alcionáceos e gorgonáceos) e de zoantídeos (Rodríguez; Cobar, 1993; Babu et al., 1997). Existem muitos exemplos de terpenoides de corais que possuem atividade biológica ou farmacológica. Rinehart et al. (1981) estudaram a atividade antiviral, antimicrobiana e 
antineoplásica dos produtos naturais extraídos de 1300 amostras de 142 espécies de cnidários; e encontraram que aproximadamente $32 \%$ dos extratos revelavam atividade antimicrobiana. Venkateswarlu et al. (1998) encontrou atividade antibacteriana de metabólitos secundários em espécies de zoantídeos; Kuramoto et al. (1998) isolaram um alcalóide com atividade inibidora da osteoporose de uma espécie do gênero Zoanthus e Grace e Jacobs (1998) descobriram atividade antiinflamatoria e analgésica num alcalóide extraído de Zoanthus sp.

As pesquisas com Produtos Naturais marinhos no Brasil tiveram início na década de 60 no Centro de Pesquisas de Produtos Naturais na Faculdade de Farmácia da UFRJ. No entanto, ainda são poucas as informações, documentadas em artigos científicos, sobre as substâncias isoladas e a atividade biológica de produtos naturais de organismos marinhos coletados ao longo dos $7500 \mathrm{~km}$ de litoral brasileiro (Pinto et al., 2002; Lhullier et al., 2006; Dresch et al., 2005). No Brasil existem alguns antecedentes de estudos químicos em cnidários, principalmente anêmonas e octocorais. Freitas e colaboradores trabalham desde a década do 80 com substancias biactivas de anêmonas-do-mar, principalmente com Bunodosoma caissarum e B. cangicum, encontrando atividade antimitótica, hemolítica, neurotóxica e antitumoral em câncer de mama humano (Freitas; Elena, 1991; Lagos et al., 2001; Malpezzi et al., 1995; Oliveira et al., 2004).

O Lapromar, Laboratório de Produtos Naturais e Ecologia Química Marina (UFF) desenvolve investigações na área da ecologia química de organismos marinhos e química de produtos naturais marinhos. Uma das líneas de estudo mais forte do laboratório é o das defesas químicas dos octocorais, a través da análise dos metabólitos secundários, principalmente de gorgónidos e do teléstido Carijoa riisei (Epifanio et al., 2000; Maia et al., 2000).

Relatos populares, principalmente de pescadores, estimulam o estudo farmacológico e toxicológico do zoantídeo Palythoa caribaeorum, já que o muco produzido pela "baba-de-boi" é usado como analgésico e antiinflamatório sendo aplicado diretamente sobre as feridas e/ou pancadas.

Levando em conta os antecedentes farmacológicos deste grupo de animais, o uso caseiro da comunidade que demonstra uma potencial ação local farmacológica e somado à quantidade abundante do zoantídeo Palythoa caribaeorum no litoral pernambucano, decidiu-se avaliar a atividade antiinflamatória e analgésica dos extratos hidroacoólicos brutos através de bioensaios em camundongos e ratos de laboratório.

\section{MATERIAL E MÉTODOS}

\section{Material animal}

Aproximadamente um quilograma da colônia de Palythoa caribaeorum foi coletado na praia de Porto de Galinhas (Pernambuco, Brasil) em fevereiro de 2003, com ajuda de faca e espátula. A amostra foi acondicionada em sacos plásticos mantidos em banho de gelo e em laboratório conservados em freezer. $\mathrm{O}$ material foi identificado pelo zoólogo Carlos Daniel Pérez da UFPE.

\section{Preparação do extrato bruto}

O extrato utilizado nos ensaios foi preparado pelo método de percolação, segundo o processo A da Farmacopéia Brasileira 2a ed. (1959) usando solução hidroalcoólica a $50 \%$. O extrato foi evaporado inicialmente em rotaevaporador e posteriormente em banho Maria com temperatura inferior a $60{ }^{\circ} \mathrm{C}$. Este extrato foi proporcionalmente diluído em solução aquosa com $2 \%$ de "tween 80" (veículo) para facilitar a administração aos animais.

\section{Animais utilizados}

Para os ensaios de toxicidade aguda e atividade analgésica foram utilizados camundongos Swiss (32 $\pm 5 \mathrm{~g}$ ), e para o teste de atividade antiinflamatória foram utilizadas ratos albinos Wistar $(210 \pm 30 \mathrm{~g})$. Os animais foram acondicionados em gaiolas com ração balanceada, água "ad libitum", em ciclo claro-escuro de 12 horas sobre temperatura constante de $22 \pm 2{ }^{\circ} \mathrm{C}$. O uso dos animais foi autorizado pela Comissão de Ética em Experimentação Animal (CEEA) da Universidade Federal de Pernambuco.

\section{Ensaio de toxicidade aguda}

O primeiro ensaio realizado foi o de toxicidade aguda em camundongos. Antes do início do ensaio, as unidades experimentais foram submetidas a jejum de 18 hs e divididas aleatoriamente em cinco grupos com três machos e três fêmeas cada um tratado "per os" com o extrato nas doses 50 mg.kg-1 v.o., 500 mg.kg-1 v.o. e i.p., $1000 \mathrm{mg} \cdot \mathrm{kg}^{-1}$ i.p. e $2000 \mathrm{mg} \cdot \mathrm{kg}^{-1}$ v.o. para a determinação da toxicidade aguda e a dose letal media $\mathrm{DL}_{50}$. Os animais foram observados durante uma hora depois da administração do extrato, e pelos 15 dias seguintes. A análise de toxicidade aguda levou a conta à observação de alterações no sistema nervoso central, sistema nervoso autônomo e atividade motora.

\section{Avaliação da Atividade Analgésica}

\section{Ensaio de Writhing}

O ensaio de "Writhing" foi realizado segundo a metodologia de Hendershot; Forsaith (1959) para detectar atividade analgésica no extrato. Foram formados cinco grupos de cinco camundongos machos cada um. Três grupos receberam o extrato $\left(200 \mathrm{mg} . \mathrm{kg}^{-1}\right.$ v.o., $400 \mathrm{mg} . \mathrm{kg}^{-1}$ v.o. e $50 \mathrm{mg}^{\mathrm{kg}}{ }^{-1}$ i.p.), um grupo recebeu veículo, servindo 
Tabela 1. Análise do efeito analgésico do extrato de Palythoa caribaeorum na síndrome de "Writhing" induzido por ácido acético em camundongos. *Resultado significativo.

\begin{tabular}{lccc}
\hline Grupos tratados & Doses e via de administração & $\begin{array}{c}\mathrm{N}^{\circ} \text { de "Writhing" em } 10 \\
\text { min }\end{array}$ & $\begin{array}{c}\text { Percentagem de inibição } \\
(\%)\end{array}$ \\
\hline Controle & \multicolumn{2}{c}{$36,0 \pm 13,58$} & $47,22 \% \%^{*}$ \\
\hline \multirow{2}{*}{ Extrato } & $200 \mathrm{mg} \cdot \mathrm{kg}^{-1}$ v.o. & $19,0 \pm 4,35$ & $37,22 \%$ \\
& $400 \mathrm{mg} \cdot \mathrm{kg}^{-1}$ v.o. & $22,6 \pm 3,91$ & $50,00 \%$ \\
\hline Fenilbutazona & $50 \mathrm{mg} \cdot \mathrm{kg}^{-1}$ i.p. & $18,0 \pm 9,76$ & $64,5 \%^{*}$ \\
\hline
\end{tabular}

Tabela 2. Análise do efeito analgésico do extrato de Palythoa caribaeorum no teste da chapa quente com camundongos. * Resultado significativo.

\begin{tabular}{l|c|c|c|c}
\hline \multirow{2}{*}{$\begin{array}{c}\text { Grupos } \\
\text { Tratados }\end{array}$} & \multirow{2}{*}{$\begin{array}{c}\text { Doses e via de } \\
\text { administração }\end{array}$} & \multicolumn{2}{|c}{ Tempo em segundos para a resposta ao estímulo depois do tratamento } \\
\cline { 3 - 5 } & & $45 ”$ & $90 ”$ & $120 ”$ \\
\hline Controle & & $9,39 \pm 4,34$ & $9,47 \pm 3,46$ & $9,62 \pm 2,17$ \\
\hline \multirow{2}{*}{ Extrato } & $200 \mathrm{mg} \cdot \mathrm{kg}^{-1}$ v.o. & $14,42 \pm 4,97$ & $11,10 \pm 6,90$ & $15,21 \pm 5,07$ \\
& $400 \mathrm{mg} \cdot \mathrm{kg}^{-1}$ v.o. & $10,27 \pm 3,52$ & $11,18 \pm 7,06$ & $12,95 \pm 3,71$ \\
& $100 \mathrm{mg} \cdot \mathrm{kg}^{-1}$ i.p. & $8,90 \pm 1,96$ & $16,52 \pm 8,10$ & $13,78 \pm 4,81$ \\
\hline Morfina & $10 \mathrm{mg} \cdot \mathrm{kg}^{-1}$ i.p. & $20,73 \pm 6,47 *$ & $17,97 \pm 8,27$ & $17,75 \pm 7,73$ \\
\hline
\end{tabular}

Tabela 3. Análise do efeito antiinflamatório do extrato de Palythoa caribaeorum no edema de pata de rato induzido por carragenina.* Resultado significativo.

\begin{tabular}{|c|c|c|c|}
\hline \multirow{2}{*}{$\begin{array}{l}\text { Grupos } \\
\text { Tratados }\end{array}$} & \multirow{2}{*}{$\begin{array}{l}\text { Doses e via de } \\
\text { Administração }\end{array}$} & \multicolumn{2}{|c|}{$\begin{array}{l}\text { Variação do volume das patas nos seguintes tempos depois da } \\
\text { aplicação da carragenina }\end{array}$} \\
\hline & & $3 \mathrm{~h}$ & $4 \mathrm{~h}$ \\
\hline Controle & & $0,96 \pm 0,2$ & $0,78 \pm 0,21$ \\
\hline Extrato & $\begin{array}{l}200 \text { mg.kg }{ }^{-1} \text { v.o. } \\
400 \text { mg.kg }{ }^{-1} \text { v.o. }\end{array}$ & $\begin{array}{l}1,09 \pm 0,24 \\
0,89 \pm 0,33\end{array}$ & $\begin{array}{l}0,82 \pm 0,16 \\
0,7 \pm 0,25\end{array}$ \\
\hline Indometacina & $10 \mathrm{mg} \cdot \mathrm{kg}^{-1} \quad$ v.o & $0,51 \pm 0,18^{*}$ & $0,31 \pm 0,12^{*}$ \\
\hline
\end{tabular}

como controle, e o último grupo recebeu tratamento analgésico com fenilbutazona $(100 \mathrm{mg} / \mathrm{Kg})$. Depois de $45^{\prime}$ da aplicação foi administrada $10 \mathrm{~mL} \cdot \mathrm{kg}^{-1}$ de solução aquosa de ácido acético i.p.; três minutos depois da aplicação do ácido foi feita a contagem de "Writhings" (contorções abdominais). A media de "Writhings" foi determinada para cada grupo, permitindo calcular a percentagem de inibição para cada dose.

\section{Ensaio da chapa quente}

Outro ensaio para medir o efeito analgésico foi o da chapa quente (Eddy; Leinbach, 1953). Cinco grupos de cinco camundongos fêmeas cada um foram tratados: 200 mg.kg-1 v.o., 400 mg. $\mathrm{kg}^{-1}$ v.o. e $100 \mathrm{mg} . \mathrm{kg}^{-1}$ i.p. de extrato, 100 mg.kg-1 de morfina i.p. e veículo. Depois de 45, 90 e 120 minutos da aplicação foram colocados em contato com a chapa quente $\left(\mathrm{T}^{\mathrm{o}}\right.$ constante de $55^{\circ} \mathrm{C}$ ) e foram registrados os segundos transcorridos antes que o animal mostrasse resposta característica ao estímulo térmico (lamber as patas anteriores e/ou "sapatear" sobre a chapa). $\mathrm{O}$ efeito produzido pelo extrato e pela morfina foi comparado com o verificado no grupo controle.

\section{Avaliação da Atividade Antiinflamatória}

\section{Ensaio de edema de pata de rato induzido por carragenina}

O último teste realizado foi o de edema de pata 
induzido por carragenina em ratos, segundo metodologia de Winter et al. (1962). Foram formados quatro grupos de oito ratos fêmeas cada um que receberam tratamentos de extrato (200 mg. $\mathrm{kg}^{-1}$ v.o. e $400 \mathrm{mg} \cdot \mathrm{kg}^{-1}$ v.o), veículo e indometacina (10 mg. $\mathrm{kg}^{-1}$ v.o.). Depois de meia hora, foi aplicado um volume de $0,1 \mathrm{~mL}$ de carragenina na região subplantar da pata traseira direita dos animais para induzir o edema. O volume da pata foi medido pletismográficamente antes da aplicação de carragenina, 3 hs y 4hs depois da aplicação.

\section{Análises estatísticas}

As medias dos dados obtidos em cada teste foram comparadas usando o teste estatístico t de Student, utilizando um nível de significância $\mathrm{P}<0,05$ (Sokal; Rohlf, 1996).

\section{RESULTADOS}

\section{Toxicidade aguda}

A administração do extrato nos ensaios de toxicidade aguda não revelou sinais deletérios envolvendo o sistema nervoso central, sistema nervoso autônomo e atividade motora. $\mathrm{O} \mathrm{DL}_{50}$ em camundongos evidenciou valores superiores a $2000 \mathrm{mg} . \mathrm{kg}^{-1}$ i.p., não observando, neste nível de dose, mortes no grupo tratado com o extrato.

\section{Ensaio de Writhing}

No ensaio de "Writhing" o extrato apresentou resultado significativo na dose de $200 \mathrm{mg} \cdot \mathrm{kg}^{-1}$ (Tabela 1)

\section{Ensaio da chapa quente em camundongos}

O teste da chapa quente aparentemente indicou atividade analgésica para o extrato (Tabela 2). Mas, não foram encontrados resultados estatisticamente significativos.

Ensaio de edema de pata de rato induzida por carragenina

O ensaio de edema de pata não indicou atividade antiinflamatória para o extrato do zoantídeo (Tabela 3 ).

\section{DISCUSÃO}

\section{Toxicidade aguda}

O gênero Palythoa se caracteriza pela presencia de una potente toxina denominada palitoxina. Esta toxina é considerada a molécula biologicamente ativa mais potente de origem marinho e a mais tóxica conhecida, sendo apenas menos venenosa que a proteína botulínica
(Kaul et al., 1974). Devido a sua elevada toxicidade $\left(\mathrm{LD}_{50}\right.$ de 0,033 a $0,45 \mu \mathrm{g} / \mathrm{kg}$ em cachorros, coelhos, macacos, hamsters e camundongos) a palitoxina tem pouca utilidade como medicamento, mais possui grande versatilidade como ferramenta bioquímica, pois afeta inúmeros processos, principalmente o transporte de íons cálcio (Wiles et al., 1974; Draijer et al., 1982).

$P$. caribaeorum é uma espécie potencialmente portadora de palitoxina, já que foi isolada anteriormente de exemplares caribenhos (Béress et al., 1983), por tal motivo esperava-se encontrar elevados índices de letalidade em doses muito baixas, mas isto não foi observado, ainda com valores superiores a $2000 \mathrm{mg} . \mathrm{kg}^{-}$ 1. Como todo metabólito secundário muitas vezes sua biosíntese está afetada por fatores do meio ambiente podendo modificar sua estrutura em condições ambientais diferentes, alterando, talvez, a parte tóxica da molécula. Igualmente, a origem da palitoxina é incerta e motivo de muitas controvérsias, alguns autores a associam aos ovos de Palythoa caribaeorum (Kimura et al., 1973), outros especulam uma origem bacteriana (Moore et al., 1982) e outros que seria uma molécula sintetizada por dinoflagelados bentônicos do gênero Ostreopsis associados ao sedimento (Onuma et al., 1999). Os resultados do presente trabalho apoiariam a idéia de que a palitoxina não é sintetizada pelo zoantídeo e sim por organismos associados ao sedimento, como bactérias ou dinoflagelados que poderiam ser, potencialmente incorporados aos tecidos destes cnidários coloniais durante seu crescimento. Esta teoria estaria altamente apoiada pelo padrão de crescimento dos indivíduos do gênero Palythoa que formam uma camada subepidérmica de sedimento que atua como sustentação (Burnett et al., 1997), já que os zoantídeos não apresentam qualquer estrutura esqueletária. A ausência de toxicidade nos exemplares estudados poderia justificar-se pela ausência dos microorganismos produtores da palitoxina no sedimento adjacente.

\section{Bioensaios}

$\mathrm{O}$ único ensaio que apresentou resultados significativos foi o de Writhing para a dose administrada por via oral de $200 \mathrm{mg} . \mathrm{kg}^{-1}$, com uma percentagem de inibição de 47,22\%, más surpreendentemente a dose administrada de $400 \mathrm{mg} \cdot \mathrm{kg}^{-1}$ só inibiu o $37,22 \%$. A dose que mostrou mais percentagem de inibição foi a de $50 \mathrm{mg} \cdot \mathrm{kg}^{-1}$, administrada por via intraperitoneal com $50 \%$ de inibições, no entanto, estatisticamente não significativas. Estes resultados indicariam que o extrato de Palythoa caribaeorum é um potencial analgésico que atuaria em doses baixas, para o qual estudos futuros serão necessários para a utilização de este animal como remédio analgésico sistêmico. Muitos analgésicos têm efeito dosedependente, o que indica que após certa concentração de administração o efeito decai ou é prejudicial; por exemplo, o Paracetamol (acetaminofeno) é um analgésico- 
antipirético que em doses acima de $1000 \mathrm{mg}$ pode induzir asma, urticária ou complicações hepáticas (Ribeiro et al., 2002), ou como os antiinflamatórios no esteroides (AINES) que são excelentes analgésicos e antipiréticos em baixas doses (Cerda; Arrau, 2002).

A ausência de toxicidade e a ação analgésica detectada nos extratos de Palythoa caribaeorum associado a grande abundância de estes animais na costa pernambucana (Fernandes, 2000) abrem um campo de investigação importante utilizando a este zoantídeo como alvo de futuras pesquisas farmacológicas. A chave do êxito de $P$. caribaeorum como fonte de metabolitos secundários com ação farmacológica está, justamente, em seu modo de crescimento. Este organismo colonial possui um crescimento vegetativo com altas taxas de regeneração (Karlson, 1988) o qual permitiria a extração do material para estudos farmacológicos sem impactar nas populações locais.

\section{AGRADECIMENTOS}

Os autores desejam expressar seu agradecimento a Secretaria de Desenvolvimento Econômico, Turismo e Meio Ambiente-SEDETMA, a Diretoria Geral de Agricultura e Meio Ambiente-DGAMA da Prefeitura Municipal de Ipojuca (Pernambuco) e a Associação dos Jangadeiros de Porto de Galinhas (AJPG) na pessoa de Junior (presidente da Associação) pelo apoio brindado nas coletas do material utilizado no presente trabalho. Agradecemos a Dra. Paula Braga Gomes (BiologiaUFRPE) pela leitura crítica do manuscrito. O trabalho contou com o financiamento da FACEPE - Fundação de Amparo à Ciência e Tecnologia do Estado de Pernambuco (Brasil) (DCR-0007-07.03/04 e MCT/ CNPqCT / INFRA/ FACEPE No 006/2003).

\section{REFERÊNCIAS}

Babu UV, Bhandari SPS, Garg HS 1997. Hariamide, a novel sulfated sphingolipid from a Zoanthus sp. of the Indian coast. J Nat Prod 60: 1307-1309.

Béréss L, Zwick HJ, Kolkenbrock PN, Kaul O, Wassermann A 1983. A method for the isolation of the caribbean palytoxin (C-PTX) from the coelenterate (zoanthid) Palythoa caribaeorum. Toxicon 21: 285-290.

Burnett WJ, Benzie JAH, Beardmore JA, Ryland JS 1997. Zoanthids (Anthozoa, Hexacorallia) from the Great Barrier Reef and Torres Strait, Australia: systematics, evolution and a key to species. Coral Reefs 16: 5568.

Cerda HO, Arrau MP2002. Manejo de dolor postoperatorio. Revista Chilena de Anestesia (on line), 31, fev. 2002. Disponível em: <http://www.socanestesia.cl/rev anestesia/0202/manejo.asp>, acessada em abril de 2006.

Correia CRD, Costa PRR, Ferreira VF 2002. Vinte e cinco anos de reações, estratégias e metodologias em química orgânica. Quim Nova 25: 82-89.

Draijer F, Suvanto P, Tesseraux I, Béréss L 1982. Mechanical studies about the action of palytoxin (Palythoa caribaeorum) on innervated and denervated rat diaphragms. Toxicon 20: 65.

Dresch RR, Haeser AS, Lerner C, Mothes B, Vozári-Hampe MM, Henriques AT 2005. deteç̧ão de atividade lectínica e atividade hemolítica em extratos de esponjas (Porifera) nativas da costa atlântica do Brasil. Rev Bras Farmacogn 15: 16-22.

Eddy NB, Leimbach D 1953. Synthetic analgesic. II. Dithienylbutenyl and dithienylbutyl amines. $J$ Pharmacol Exp Ther 107: 385-393.

Epifanio RA, Maia LF, Fenical W 2000. Chemical defenses of the endemic brazilian gorgonian Lophogorgia violacea Pallas (Octocorallia, Gorgonacea). J Braz Chem Soc 11: 584-591.

Farmacopéia dos Estados Unidos do Brasil. 1959. São Paulo: Siqueira, 2a ed.

Fernandes MLB 2000. Avaliação de dois ambientes recifais do litoral de Pernambuco, através das suas macro e megafaunas incrustantes e sedentárias. São Paulo, 179p. Tese de Doutorado - Universidade de São Paulo.

Freitas JC, Elena LA 1991. Hemolytic activity of the nematocyst venom from the sea anemone Bunodosoma caissarum. Braz J Med Biol Res 24: 1245-1249.

Grace KJS, Jacobs RS 1998. The anti-inflamatory and analgesic activies of zoanthamine, a new structural alkaloid from the toxic colonial zoanthid, Zoanthus sp. FASEB $J$ 2: A1109.

Hendershot LC, Forsaith S 1959. Antagonison in the mouse by weak analgesics and non-analgesics. $J$ Pharmacol Exp Ther 125: 237-240.

Karlson RH 1988. Size-dependent growth in two zoanthid species: A contrast in clonal strategies. Ecology 69: 1219-1232.

Kaul PN, Farmer MR, Ciereszko LS 1974. Pharmacology of palytoxin: The most potent marine toxin known. Proc West Pharmacol Soc 17: 294.

Kimura S, Hashimoto Y, Yamazato K 1973. Toxicity of the zoanthid Palythoa tuberculosa. Toxicon 10: 611-617.

Kuramoto M, Hayashi K, Yamaguchi K, Yada M, Tsuji T, Uemura D 1998. Structure-activity relationship of norzoanthamine exhibiting significant inhibition of osteoporosis. Bull Chem Soc Jpn 71: 771-779.

Lagos P, Freitas JC, Duran RC, Silveira R 2001. Identification of hemolytic and neurotoxic activities in the venom of the sea anemone Bunodosoma cangicum. Braz J Med Biol Res 34: 895-902.

Lhullier C, Horta PA, Falkenberg M 2006. Avaliação de extratos de macroalgas bênticas do litoral catarinense utilizando o teste de letalidade para Artemia salina. Rev Bras Farmacogn 16: 158-163.

Maia LF, Epifanio RA, Fenical W 2000. New cytotoxic glicosides from the octocoral Carijoa (Telesto) riisei (Telestacea, Octocorallia). J Nat Prod 63: 14271430.

Malpezzi EL, Matsui DH, Groote SC, Freitas GM, Santelli GM, Fernandes JB 1995. Antitumoral activity in an organic extract of the sea anemone Bunodosoma caissarum. Toxicon 33: 291.

Moore RE, Helfrich P, Patterson GML 1982. The deadly seaweed of Hana. Oceanus 25: 54.

Oliveira JS, Redaelli E, Zaharenko AJ, Casslini RR, Konno K, Pimenta DC, Freitas JC, Clare JJ, Wanke E 2004. 
Binding secificity of sea anemone toxins to nav 1.11.6 sodium channels unexpected contributions from differences in the IV/S3-S4 outer loop. J Biol Chem 279: 33323-33325.

Onuma Y, Masayuki S, Ukena T, Roux J, Chanteau S, Rasolofonirina N, Ratsimaloto M, Naoki H, Yasumamoto $\mathrm{T}$ 1999. Identification of putative palytoxin as the cause of clupeotoxism. Toxicon 37: 55-65.

Pérez CD 1999. Taxonomía, distribución y diversidad de los Pennatulacea, Gorgonacea y Alcyonacea del Mar Epicontinental Argentino y zonas de influencia. Mar del Plata, Argentina, xiii $+254 \mathrm{p}$. Tese de Doutorado - Universidad Nacional de Mar del Plata, Argentina.

Pinto AC, Silva DHS, Bolzani VD, Lopes NP, Epifanio RD 2002. Current status, challenges and trends on natural products in Brazil. Quim Nova 25: 45-61.

Ribeiro S, Schmidt AP, Schmidt SRG 2002. O uso de opióides no tratamento da dor crônica não oncológica: o papel da metadona. Rev Bras Anestesiol 52: 644-651.

Rinehart KL, Shaw PD, Shield LS, Gloer JB, Harbour GC, Koker MES, Samain D, Schwartz RE, Tymiak AA, Weller DL, Carter GT, Munro MH, Hughes RG, Renis HE, Swynenberg EB, Stringfellow DA, Vavra JJ, Coats JH, Zurenko GE, Kuentzel SL, Li LH, Bakus GJ, Brusca RC, Craft LL, Yong DN, Conner JL 1981. Marine natural products as sources of antiviral, antimicrobial and antineoplastic agents. Pure Appl Chem 53: 795-817.

Rodriguez AD, Cobar OM 1993. Structures and bioactivities of new asbestinim diterpenoids from the Caribbean gorgonian octocoral Briareum asbestinum. Tetrahedron 49: 319-328.

Sokal RR, Rohlf FJ 1996. Biometry. New York: W. H. Feeman and Company.

Venkateswarlu Y, Reddy NS, Ramesh P, Reddy PS, Jamil K 1998. Chemical reduction of zoanthamine and evaluation of antibacterial activity. Heterocycl Commun 4: 575-580.

Weinheimer A, Spraggins R 1969. The occurrence of two new prostaglandin derivates (15-epi-PGA2 and its acetate, methyl ester) in the gorgonian Plexaura homomalla. Chemistry of Coelenterates, XV. Tetrahedron Lett 59: 5185-5188.

Wiles JS, Vick JA, Christensen MK 1974. Toxicological evaluation of palytoxin in several animal species. Toxicon 12: 427-433.

Winter CA, Risley EA, Nuss GW 1962. Carrageenin-induced edema in hing paw of the rat as an assay for antiinflamatory drugs. Proc Soc Exp Biol Med 111: 544-547. 